\title{
Concept of a low energy electron-positron collider for dimuonium study
}

\section{A. Bogomyagkov and V. Druzhinin and E. Levichev and A. Milstein and S. Sinyatkin} Budker Institute of Nuclear Physics, Novosibirsk

E-mail: A.V.Bogomyagkov@inp.nsk.su, V.P.Druzhinin@inp.nsk.su,

E.B.Levichev@inp.nsk.su, A.I.Milstein@inp.nsk.su,

S.V.Sinyatkin@inp.nsk.su

We discuss a low energy collider for production of the not yet discovered $\mu^{+} \mu^{-}$bound state (dimuonium). In our design we follow the Brodsky-Lebed proposal of the large-crossing-angle $e^{+} e^{-}$intersection, when the dimuonium carries non-zero momentum and decays to $e^{+} e^{-}$pair far away from the beam collision region. The latter provides effective suppression of the Bhabha scattering background. We study experimental constraints and following requirements for the collider development. A preliminary layout is considered, the main parameters are obtained. The expected peak luminosity at the $\mu^{+} \mu^{-}$production threshold is $8 \times 10^{31} \mathrm{~cm}^{-2} \mathrm{~s}^{-1}$. The same machine can be used for high statistic study of hadronic processes $\left(e^{+} e^{-} \rightarrow \pi^{+} \pi^{-}, \pi^{+} \pi^{-} \pi^{0}, \pi^{0} \gamma\right.$ etc.) and search for rare processes in the center-of-mass energy range below $960 \mathrm{MeV}$.

The European Physical Society Conference on High Energy Physics

5-12 July, 2017

Venice 


\section{Introduction}

Currently some authors consider a lepton atom $\left(\mu^{+} \mu^{-}\right)$(dimuonium, bimuonium or true muonium) as a strong candidate for casting light on disagreement between the anomalous magnetic moment measurement and Standard Model prediction, the muonic hydrogen proton's charge radius discrepancy, etc. [2,3]. Even if this expectation will not come true, a discovery of a new lepton bound state, which has not been observed yet contrary to positronium $\left(e^{+} e^{-}\right)$and muonium $\left(\mu^{+} e^{-}\right)$, and its study is by far a challenging and interesting scientific enterprise. Like positronium, dimuonium is a Bohr atom and its study (including transition spectroscopy, lifetime precise measurement, etc.) allows verifying QED and quantum mechanics computations with great accuracy. In such a research, $\left(\mu^{+} \mu^{-}\right)$has higher new-physics reach potential in comparison with other exotic atoms. It has larger reduced mass $\mu=m_{\mu} / 2$ than $\left(e^{+} e^{-}\right)$and $\left(\mu^{+} e^{-}\right)$, for which $\mu \approx m_{e}$; therefore, it is considerably more compact (the dimuonium Bohr radius is 200 times smaller than the positronium one). In contrast to $\left(p e^{-}\right)$and $\left(p \mu^{-}\right)$systems, $\left(\mu^{+} \mu^{-}\right)$experiences no strong interaction obscuring exploration of tiny QED effects.

We propose a preliminary design of the electron-positron collider for $\left(\mu^{+} \mu^{-}\right)$search and study. Low beam energy $E_{b} \approx 400 \mathrm{MeV}$ results in a compact machine, inexpensive in both manufacture and maintenance. Large crossing angle of the intersecting beams induces a non-zero dimuonium momentum which is a distinct feature of our design (originally proposed in [4]). In this case, the $\left(\mu^{+} \mu^{-}\right)$production and decay points are spatially separated along the beams directions bisector (for equal beam energies). The distance between the atom formation and its annihilation into $e^{+} e^{-}$ pair effectively suppresses the primary beams elastic scattering background and allows to detect unambiguously detect the dimuonium decay.

Besides the $\left(\mu^{+} \mu^{-}\right)$research, the beam energy and collider configuration allow studying such processes as $e^{+} e^{-} \rightarrow$ pions and $e^{+} e^{-} \rightarrow \eta, \eta^{\prime}$ expanding substantially the proposed facility experimental program.

\section{Dimuonium}

Dimuonium [5] is one of the simplest hydrogen-like and similar to positronium. Unlike positronium dimuonium annihilates into $e^{+} e^{-}$pair, and this makes its formation and spectrum more sensitive to vacuum polarization effects caused by virtual electron-positron pairs production. The $\left(\mu^{+} \mu^{-}\right)$atom exists in two spin states: paradimuonium, which is a singlet with total muonantimuon spin $S=0$, and orthodimuonium (triplet $S=1$ ). For paradimuonium ground state with the dominant decay mode $1^{1} S_{0} \rightarrow \gamma \gamma$ the lifetime is $0.602 \mathrm{ps}$ while for orthodimuonium it is $1.81 \mathrm{ps}$ (dominant decay is $1^{3} S_{1} \rightarrow e^{+} e^{-}$). The lifetime of muon weak decay is $2 \mu \mathrm{s}$, and thus, dimuonium is a unique metastable system available for precision QED tests.

Peak cross section of $\left(\mu^{+} \mu^{-}\right)$production in the electron-positron collision for exact resonance condition [1] (the beam energy $E_{b}=m_{\mu}-\Delta_{\mu \mu} / 2, m_{\mu}$ is muon mass and $\Delta_{\mu \mu} \approx 1.41 \mathrm{keV}$ is the binding energy) is $\sigma(\mu \mu)=3.3 \cdot 10^{-25} / \mathrm{n}^{3} \mathrm{~cm}^{-2}$. Radiative corrections reduce the cross section. For instance, the reduction for the ground state is $\sigma_{1 R}(\mu \mu)=0.27 \sigma_{\mu \mu}$. 
However, detailed simulation of the $\left(\mu^{+} \mu^{-}\right)$production rate with account for the beam energy and spatial distribution recovers the cross section reduction due to the radiative corrections (within $10 \%$ accuracy), thus for dimuonium production rate estimation we use peak cross section.

\section{Large angle beam crossing}

Using results from [6], geometry from Figure 1 and assuming Gaussian beam divergence and energy spread we derived mean and r.m.s values of invariant mass $\langle M\rangle_{x^{\prime}, y^{\prime}, \delta}$ and $\sigma_{M}$ for collision with crossing angle $\theta$

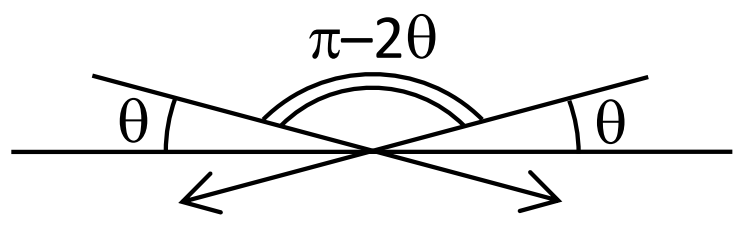

Figure 1: Electron positron collision at arbitrary angle $\theta$.

$$
\begin{aligned}
\sigma_{M}^{2} & =2 E_{0}^{2}\left[\sigma_{\delta}^{2} \cos (\theta)^{2}+\sigma_{p x}^{2} \sin (\theta)^{2}\right], \\
\langle M\rangle_{x^{\prime}, y^{\prime}, \delta} & =2 E_{0} \cos (\theta)-\frac{1}{2} E_{0} \sigma_{\delta}^{2} \cos (\theta)-\frac{1}{2} E_{0} \sigma_{p x}^{2} \cos (\theta)-\frac{1}{2} E_{0} \sigma_{p y}^{2} \frac{\cos (2 \theta)}{\cos (\theta)},
\end{aligned}
$$

where $E_{0}$ is mean energy of each beam, $\sigma_{\delta}, \sigma_{p x}$ and $\sigma_{p y}$ are r.m.s values of beam energy spread and horizontal and vertical divergences. Luminosity [6] and production rate are

$$
\begin{aligned}
\mathscr{L}_{0} & =\frac{N_{1} N_{2}}{4 \pi \sigma_{x} \sigma_{y} \sqrt{1+\varphi^{2}}} f_{0} N_{\text {bunch }}, \\
\dot{N} & =\frac{\Gamma_{\mu \mu} \sigma_{\mu \mu} \mathscr{L}_{0}}{2 \sqrt{\pi} \sigma_{M}},
\end{aligned}
$$

where $\varphi=\sigma_{z} \tan \theta / \sigma_{x}$ is Piwinski angle, $N_{1,2}$ is bunch population, $\sigma_{x, y}$ is (horizontal, vertical) r.m.s. beam size, $f_{0}$ is revolution frequency, $N_{\text {bunch }}$ is number of bunches, and we used approximate expression for cross-section $\sigma\left(x_{1,2}^{\prime}, y_{1,2}^{\prime}, \delta_{1,2}\right)=\Gamma_{\mu \mu} \sigma_{\mu \mu} \cdot \delta\left(M\left(x_{1,2}^{\prime}, y_{1,2}^{\prime}, \delta_{1,2}\right)-M_{\mu \mu}\right)$.

\section{Background and collider parameters}

The main source of the background is elastic $e^{+} e^{-} \rightarrow e^{+} e^{-}$scattering with cross section $\sigma_{\text {Bhabha }}=22000 \mathrm{nb}$. Background suppression is possible if dimuonium decay point is separated from origin point; dimuonium lifetime at rest $\tau_{0 \mu \mu}=\hbar / \Gamma_{\mu \mu}$ provides separation $l=c \tau_{0 \mu \mu} \beta_{\mu \mu} \gamma_{\mu \mu}=$ $c \tau_{0 \mu \mu} \tan \theta=c \hbar \tan \theta / \Gamma_{\mu \mu}$. The signal to background ratio then is

$$
\frac{\dot{N}_{\mu \mu}}{\dot{N}_{e e}} \propto \frac{\exp \left[\frac{\left(c \tau_{0 \mu \mu}\right)^{2} \sin ^{2} \theta}{\sigma_{x}^{2}}\right]}{\sqrt{\sigma_{\delta}^{2} \cos ^{2} \theta+\left(\sigma_{x}^{2} / \beta_{x}^{2}\right) \sin ^{2} \theta}},
$$


Table 1: Collider parameters.

\begin{tabular}{l|c}
\hline Beam energy $(\mathrm{MeV})$ & 408 \\
\hline Crossing angle $\theta(\mathrm{deg})$ & 75 \\
\hline Circumference $(\mathrm{m})$ & 23 \\
\hline Number of particles / bunch current $(\mathrm{mA})$ & $3.5 \times 10^{10} / 73$ \\
\hline Revolution frequency $(\mathrm{MHz}) /$ period $(\mathrm{ns})$ & $13.04 / 76.7$ \\
\hline Synchrotron tune & 0.0171 \\
\hline Momentum compaction $\alpha$ & 0.064 \\
\hline Damping times hor./vert./long. $(\mathrm{ms})$ & $17.3 / 27.3 / 22.1$ \\
\hline Hor. emittance no IBS/with IBS $(\mathrm{nm})$ & $26 / 90$ \\
\hline Energy spread no IBS/with IBS $\left(10^{-4}\right)$ & $4 / 8.4$ \\
\hline Bunch length no IBS/with IBS $(\mathrm{mm})$ & $5.4 / 11.6$ \\
\hline Coupling & $0.3 \%$ \\
\hline Angular spread $\sigma_{x^{\prime}}^{*}\left(10^{-4}\right)$ & 6.7 \\
\hline$\sigma_{M}(\mathrm{keV})$ & 390 \\
\hline$\beta_{x}^{*} / \beta_{y}^{*}(\mathrm{~mm})$ & $200 / 2$ \\
\hline$\sigma_{x}^{*} / \sigma_{y}^{*}(\mu \mathrm{m})$ & $130 / 0.7$ \\
\hline Luminosity $(1$ bunch $)\left(\mathrm{cm}^{-2} \mathrm{~s}^{-1}\right)$ & $4 \times 10^{30}$ \\
\hline Luminosity $(20$ bunches $)\left(\mathrm{cm}^{-2} \mathrm{~s}^{-1}\right)$ & $8 \times 10^{31}$ \\
\hline
\end{tabular}

Table 2: Dimuonium production rate estimation for collider parameters from Table 1.

\begin{tabular}{l|c|c}
\hline$\left(\mu^{+} \mu^{-}\right)$events & per hour & per 4 months \\
\hline All $(1 S / 2 S / 3 S)$ & $65 / 8.1 / 2.4$ & $187000 / 23000 / 6900$ \\
\hline$\Delta L>2 \mathrm{~mm}$ & $21 / 7 / 2.3$ & $59000 / 20000 / 6600$ \\
\hline
\end{tabular}

where $\beta_{x}$ is horizontal beta function value at interaction point and $\sigma_{p x}^{2}=\sigma_{x}^{2} / \beta_{x}^{2}$. Higher signal to background ratio requires large crossing angle and small horizontal beam size, small beam divergence. However, large crossing angle increases beam energy, enlarges invariant mass resolution and decreases luminosity. Balance of these considerations allows to define collider parameters shown in Table 1 with production rate shown in Table 2.

Our parameters provide $l>2 \mathrm{~mm}$ and suppress the particle density in the beam tail (and the elastic scattering background rate) by 4.5 standard deviations for the ground state $1^{3} S_{1}$.

Collider consists of two symmetric rings with two collision points. Figure 2 and figure 3 demonstrate $e^{+} e^{-}$orbits and lattice functions.

\section{Conclusion}

We have considered preliminary design of the electron-positron collider for production and study of the $\left(\mu^{+} \mu^{-}\right)$bound state and capable to perform other experiments with high luminosity in the center-of-mass energy range $E_{C M} \approx 500 \div 1000 \mathrm{MeV}$. Two rings collider with large crossing angle is quite compact, the ring orbit length $23 \mathrm{~m}$, and not expensive in development and maintenance. Despite many challenging issues still needing investigation (dynamic aperture, collective 


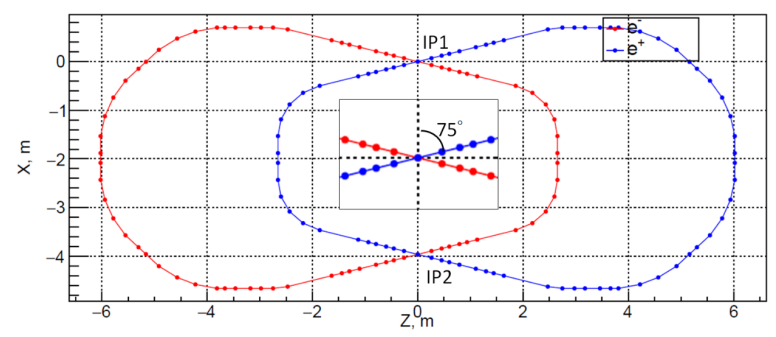

Figure 2: Geometry of the collider rings.

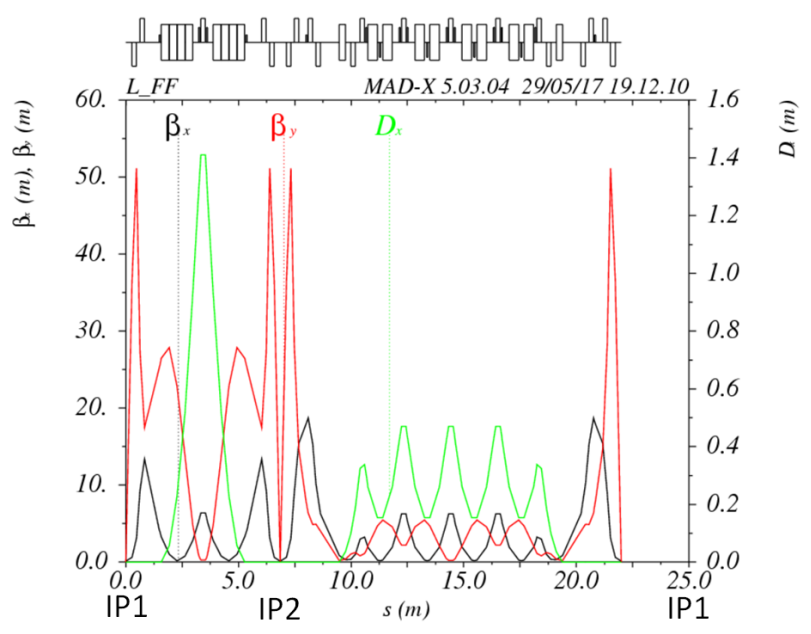

Figure 3: Collider lattice functions.

effects, magnet and vacuum systems design, etc.); the project seems interesting for both fundamental research and collider technologies development.

Collider performance and dimuonium production rate allow not only detection of new leptonic atoms for the first time but also study $\left(\mu^{+} \mu^{-}\right)$properties (including precise measurement of the lifetime, spectroscopic experiments, atoms passing through a thin foil, etc.).

\section{References}

[1] V.N.Baier and V.S.Synakh, Bimuonium production in electron-positron collisions, SOVIET PHYSICS JETP, 14, N5, 1962, pp.1122-1125.

[2] H. Lamm, True muonium: the atom that has it all, [arXiv 1509.09306v1], 30 Sep 2016.

[3] H. Lamm and R.F. Lebed, True Muonium $\left(\mu^{+} \mu^{-}\right)$on the Light Front, [hep-ph/1311.3245v3].

[4] S.J. Brodsky and R.F. Lebed, Production of the Smallest QED Atom: True Muonium $\left(\mu^{+} \mu^{-}\right)$, Phys. Rev. Lett., 102:213401, 2009. 
[5] H. Lamm, True muonium on the light front, PhD Dissertation, Arizona State University, May 2016, 115 pages.

[6] A. Bogomyagkov and E. Levichev, Collision monochromatization in $e^{+} e^{-}$colliders, Phys. Rev. Accel. Beams 20, 051001, May 2017. 